\title{
Occupational exposure to petroleum-based and oxygenated solvents and hypopharyngeal and laryngeal cancer in France: the ICARE study
}

Christine Barul ${ }^{1,2}$, Matthieu Carton ${ }^{3}$, Loredana Radoi ${ }^{4,5}$, Gwenn Menvielle ${ }^{6}$, Corinne Pilorget ${ }^{7,8}$, Simona Bara ${ }^{9}$, Isabelle Stücker ${ }^{4}$, Danièle Luce ${ }^{1 *}$ (D) ICARE study group

\begin{abstract}
Background: To examine associations between occupational exposure to petroleum-based and oxygenated solvents and the risk of hypopharyngeal and laryngeal cancer.

Methods: ICARE is a large, frequency-matched population-based case-control study conducted in France. Lifetime occupational history, tobacco smoking and alcohol consumption were collected. Analyses were restricted to men and included 383 cases of hypopharyngeal cancer, 454 cases of laryngeal cancer, and 2780 controls. Job-exposure matrices were used to assess exposure to five petroleum-based solvents (benzene; gasoline; white spirits; diesel, fuels and kerosene; special petroleum products) and to five oxygenated solvents (alcohols; ketones and esters; ethylene glycol; diethyl ether; tetrahydrofuran). Odds ratios (ORs) adjusted for smoking, alcohol drinking and other potential confounders and 95\% confidence intervals $(\mathrm{Cl})$ were estimated with unconditional logistic models.

Results: No significant association was found between hypopharyngeal or laryngeal cancer risk and exposure to the solvents under study. Non-significantly elevated risks of hypopharyngeal cancer were found in men exposed to high cumulative levels of white spirits $(\mathrm{OR}=1.46 ; 95 \% \mathrm{Cl}: 0.88-2.43)$ and tetrahydrofuran $(\mathrm{OR}=2.63 ; 95 \mathrm{Cl} \%: 0.55-12.65)$, with some indication of a dose-response relationship ( $p$ for trend: 0.09 and 0.07 respectively).

Conclusion: This study provides weak evidence for an association between hypopharyngeal cancer and exposure to white spirits and tetrahydrofuran, and overall does not suggest a substantial role of exposure to petroleum-based or oxygenated solvents in hypopharyngeal or laryngeal cancer risk.
\end{abstract}

Keywords: Solvents, Occupational exposure, Cancer, Larynx, Hypopharynx

\section{Background}

Despite a decrease in the last decades, incidence of hypopharyngeal cancer and laryngeal cancer in France among men remains among the highest in Europe, with annual incidence rates of about 5/100,000 and 7/100,000 respectively [1]. Tobacco smoking and alcohol consumption are the major risk factors [2], their joint effect being at least multiplicative [3].

\footnotetext{
* Correspondence: daniele.luce@inserm.fr

1 Univ Rennes, Inserm, EHESP, Irset (Institut de recherche en santé, environnement et travail)-UMR_S 1085, Pointe-à-Pitre F-97110, France

Full list of author information is available at the end of the article
}

Several occupational exposures are also known or suspected to be associated with these cancers. Exposure to strong acid mists [4] and to asbestos [5] are recognized risk factors for laryngeal cancer, and there is also some evidence that exposure to asbestos increases the risk of hypopharyngeal cancer [5]. Other possible occupational risk factors include exposure to polycyclic aromatic hydrocarbons, engine exhausts, and solvents [6]. Exposure to solvents in general was found to be associated with an increased risk of laryngeal or hypopharyngeal cancer in several studies [7-9], but the role of specific solvents was rarely investigated. 
We previously examined the role of chlorinated solvents in head and neck cancer risk, and found an association between exposure to perchloroethylene and laryngeal cancer [10]. In our study population, increased risks of laryngeal and/or hypopharyngeal cancer were also observed among building caretakers, cleaners, farm workers, toolmakers, rubber and plastic workers, who may be exposed to other types of solvents [11]. Therefore, our objective in this study was to examine the associations between exposure to petroleum-based and oxygenated solvents and the risk of hypopharyngeal and laryngeal cancers.

\section{Methods}

\section{Study design and population}

The present study is based on data from the ICARE study, a French multicenter population-based casecontrol study, conducted between 2001 and 2007 in ten geographical areas covered by a cancer registry. Eligible cases were patients aged between 18 and 75 years, with histologically confirmed tumor of the oral cavity, pharynx, sinonasal cavities and larynx first diagnosed during the study period.

Population controls were selected in the same geographical areas using incidence density sampling, with frequency-matching by gender and age $(<40,40-54$, $55-64, \geq 65$ years old). A further stratification was performed to make controls comparable to the general population on socioeconomic status. The participation rates were $82.5 \%$ among cases and $80.6 \%$ among controls. Details about the study design have been described elsewhere [12]. The present study was restricted to squamous cell carcinomas of the larynx (International Classification of Diseases for Oncology 3rd revision codes: C32) and hypopharynx (C12-C13). Analyses were restricted to men and women were analyzed separately [13]. Overall, 383 cases of hypopharyngeal cancer and 454 cases of laryngeal cancer, and 2780 controls were included.

\section{Data collection}

Standardized questionnaires were used by trained interviewers during face-to-face interviews to collect data. Those included sociodemographic characteristics, smoking and alcohol consumption histories, and a detailed lifetime occupational history, with a description of each job held for at least one month.

Trained coders blinded to case-control status coded occupations and industries, according to the International Standard Classification of Occupations (ISCO) [14] and the French Nomenclature of Activities (NAF) [15].

\section{Exposure assessment}

Exposures to five petroleum-based solvents (benzene; gasoline; white spirits and other light aromatic mixtures; diesel, fuels and kerosene; special petroleum products) and five oxygenated solvents (alcohols; ketones and esters; ethylene glycol; diethyl ether; tetrahydrofuran) were assessed by job-exposures matrices (JEMs) developed for the French population in the context of the Matgéné program [16]. The JEMs assessed both inhalation and dermal exposures, with no distinction in exposure rating by route of exposure. Inhalation is, however, the main route of exposure for the solvents under study.

For each combination of ISCO and NAF codes, three indices of exposure were provided by the JEMs: (i) probability of exposure expressed as the percentage of exposed workers; (ii) intensity of exposure; and (iii) frequency of exposure as a percentage of working time. For these three indices, different categories were used according to the solvent (See Additional file 1). Exposure indices were provided for different calendar periods to take into account variations due to changes in exposure over time. Specific JEMs were also used to assess exposure to asbestos [17] and perchloroethylene [10]. Exposure to strong inorganic acids was obtained for each job from specific questions in the questionnaire.

Two variables were computed by linking lifetime occupational history with these indices: 'ever/never' exposed ('ever' defined as having worked in at least one job with probability of exposure greater than zero), and the Cumulative Exposure Index (CEI). CEIs were the results of summation over the entire work history of the product of exposure probability, frequency, intensity and duration of each job period, using the central value of the classes. CEIs were categorized in four categories: 'never exposed', and three categories according to the percentiles of the distribution among exposed controls (low: < 50th; medium: 50th-90th; high: > 90th). We estimated lifetime exposure prevalence to the various solvents as the mean of the maximum probability of exposure of each subject over his working life, using the central values of the classes.

\section{Other variables}

Analyses were adjusted for age at interview in categories $(<40,40-49,50-59 ; 60-69, \geq 70$ years), residence area, alcohol consumption in categories $(\leq 0.03,0.04-2.00,2$. 01-4.99, 5.00-7.99, 8.00-11.99, $\geq 12$ glasses/day), smoking status (never; former: time since stopping smoking > 2 years at the interview; current), daily amount of tobacco in categories $(1-10,11-20,20-25,>25$ g/day), duration of tobacco smoking in categories $(1-20,21-30$, $31-40,>40$ years), and cumulative asbestos exposure in 4 categories (never exposed, and tertiles of the distribution among exposed controls). 


\section{Statistical analysis}

Adjusted odds ratios (ORs) and corresponding 95\% confidence intervals $(95 \% \mathrm{CI})$ were obtained by use of multivariable unconditional logistic regression models. Main analyses were performed separately for each solvent. Tests for linear trends were performed by modelling the median of each category as a continuous variable. We also examined the risks associated with joint exposure to several solvents by families of solvents (i) petroleumbased, (ii) oxygenated solvents. These analyses were restricted to combinations of solvents with at least 10 exposed cases.

In additional analyses we also adjusted for socioeconomic status, assessed by occupational class of the longest job held. As we previously found that exposure to perchloroethylene [10] was associated with laryngeal cancer, adjustment for this exposure was also performed but did not modify ORs estimates. Similarly, adjustment for self-reported exposure to strong inorganic acids did not change the ORs. Therefore, strong inorganic acids and perchloroethylene were not included in the models presented here.

\section{Results}

Table 1 shows the main characteristics of cases and controls, as well as lifetime prevalence of exposure to the various solvents. Cases of hypopharyngeal and laryngeal cancers were generally older, more often blue collar workers, daily drank more alcohol and were more often smokers than controls. Exposure to petroleum-based solvents was relatively frequent, and exposure prevalences were higher in cases than in controls. Exposure prevalences were lower for oxygenated solvents, and were roughly similar in cases and in controls.

The solvents studied are used as cleaners, degreasers and reagents in varied industrial processes, and are used in a number of formulations such as paints, adhesives, inks and dyes, dry cleaning solutions, pesticides, fuels, cosmetics and pharmaceuticals.

In our study population, men exposed to petroleum solvents were mainly employed as machinery fitters, and to a lesser extent as transport drivers (diesel/fuels/kerosene, gasoline) and construction workers (white spirits). Machinery fitters was the most frequent occupation among men exposed to ketones, esters, and ethylene glycol. Men exposed to ether and alcohols were mostly employed as medical workers. Exposure to tetrahydrofuran occurred primarily among plumbers and welders, due to the use of PVC pipe glues (data not shown).

Exposures to the solvents under study were correlated. The stronger correlations were found between exposures to benzene and white spirits $(r=0.62)$, gasoline and diesel/fuels/kerosene $(r=0.69)$ and ketones/esters and alcohols $(r=0.64)$ (see Additional file 2).
Table 1 Main characteristics of cases and controls

\begin{tabular}{|c|c|c|c|c|c|c|}
\hline & \multicolumn{2}{|c|}{$\begin{array}{l}\text { Hypopharyngeal } \\
\text { cancer } \\
n=383\end{array}$} & \multicolumn{2}{|c|}{$\begin{array}{l}\text { Laryngeal } \\
\text { cancer } \\
n=454\end{array}$} & \multicolumn{2}{|c|}{$\begin{array}{l}\text { Controls } \\
n=2780\end{array}$} \\
\hline & $\mathrm{n}$ & $\%$ & $\mathrm{n}$ & $\%$ & $\mathrm{n}$ & $\%$ \\
\hline \multicolumn{7}{|l|}{ Age } \\
\hline$<40$ & 0 & 0.0 & 5 & 1.10 & 76 & 2.7 \\
\hline $40-49$ & 51 & 13.3 & 56 & 12.3 & 555 & 20.0 \\
\hline $50-59$ & 164 & 42.8 & 175 & 38.6 & 825 & 29.7 \\
\hline $60-69$ & 118 & 30.8 & 147 & 32.4 & 939 & 33.8 \\
\hline$\geq 70$ & 50 & 13.1 & 71 & 15.6 & 385 & 13.9 \\
\hline \multicolumn{7}{|l|}{ Socioeconomic status } \\
\hline Farmers & 8 & 2.1 & 18 & 4.0 & 168 & 6.0 \\
\hline Self-employed workers & 25 & 6.6 & 34 & 7.5 & 152 & 5.5 \\
\hline Managers & 21 & 5.5 & 34 & 7.5 & 544 & 19.6 \\
\hline Intermediate occupations & 32 & 8.4 & 55 & 12.2 & 564 & 20.3 \\
\hline Employees & 40 & 10.4 & 45 & 10.0 & 297 & 10.7 \\
\hline Blue collar workers & 252 & 65.8 & 265 & 58.8 & 1053 & 37.9 \\
\hline \multicolumn{7}{|l|}{ Alcohol consumption, glasses/day } \\
\hline$\leq 0.03$ & 11 & 2.9 & 19 & 4.2 & 206 & 7.5 \\
\hline $0.04-2.00$ & 34 & 8.9 & 70 & 15.4 & 1190 & 42.8 \\
\hline $2.01-4.99$ & 89 & 23.2 & 110 & 24.2 & 849 & 30.5 \\
\hline $5.00-7.99$ & 78 & 20.4 & 108 & 23.8 & 305 & 10.9 \\
\hline $8.00-11.99$ & 82 & 21.4 & 74 & 16.3 & 134 & 4.8 \\
\hline$\geq 12$ & 70 & 18.3 & 54 & 11.9 & 73 & 2.6 \\
\hline \multicolumn{7}{|l|}{ Smoking status } \\
\hline Never & 3 & 0.8 & 13 & 2.9 & 753 & 27.1 \\
\hline Former & 121 & 31.6 & 122 & 26.9 & 1271 & 45.7 \\
\hline Current & 256 & 66.9 & 317 & 69.8 & 751 & 27.0 \\
\hline \multicolumn{7}{|l|}{ Lifetime exposure prevalence } \\
\hline \multicolumn{7}{|l|}{ Petroleum-based solvents } \\
\hline Benzene & & 16.9 & & 15.6 & & 11.7 \\
\hline Gasoline & & 11.6 & & 11.1 & & 8.7 \\
\hline $\begin{array}{l}\text { Special petroleum-based } \\
\text { products }\end{array}$ & & 3.6 & & 2.6 & & 2.5 \\
\hline Diesel, fuels and kerosene & & 20.0 & & 18.4 & & 14.4 \\
\hline White spirits & & 21.3 & & 18.7 & & 14.4 \\
\hline \multicolumn{7}{|l|}{ Oxygenated solvents } \\
\hline Ketones and esters & & 13.5 & & 11.8 & & 9.5 \\
\hline Alcohols & & 12.2 & & 11.5 & & 12.9 \\
\hline Diethyl ether & & 0.4 & & 0.6 & & 1.4 \\
\hline Ethylene glycol & & 6.5 & & 7.0 & & 6.1 \\
\hline Tetrahydrofuran & & 1.6 & & 1.0 & & 0.7 \\
\hline
\end{tabular}

Table 2 reports the associations between occupational exposure to petroleum-based solvents and hypopharyngeal and laryngeal cancer risk. Overall, no significant association was found. For hypopharyngeal cancer, the 
Table 2 Association between hypopharyngeal and laryngeal cancers and occupational exposure to petroleum-based solvents

\begin{tabular}{|c|c|c|c|c|c|c|c|}
\hline \multirow[b]{2}{*}{ Petroleum based solvents } & \multirow{2}{*}{$\begin{array}{l}\text { Controls } \\
\mathrm{n}\end{array}$} & \multicolumn{3}{|c|}{ Hypopharynx } & \multicolumn{3}{|c|}{ Larynx } \\
\hline & & $\bar{n}$ & $\mathrm{OR}^{\mathrm{a}}$ & {$[95 \% \mathrm{Cl}]$} & $\overline{\mathrm{n}}$ & $\mathrm{OR}^{\mathrm{a}}$ & {$[95 \% \mathrm{Cl}]$} \\
\hline \multicolumn{8}{|l|}{ Benzene } \\
\hline Never & 2120 & 237 & - & & 314 & - & \\
\hline Ever & 552 & 102 & 1.07 & {$[0.80-1.45]$} & 109 & 0.94 & {$[0.71-1.24]$} \\
\hline \multicolumn{8}{|l|}{ CEI } \\
\hline Low & 279 & 51 & 1.15 & {$[0.78-1.69]$} & 46 & 0.81 & {$[0.55-1.18]$} \\
\hline Medium & 220 & 39 & 1.02 & {$[0.67-1.57]$} & 56 & 1.27 & {$[0.87-1.84]$} \\
\hline High & 53 & 12 & 0.9 & {$[0.43-1.89]$} & 7 & 0.42 & {$[0.18-1.01]$} \\
\hline$p$ for trend & & & & 0.98 & & & 0.21 \\
\hline \multicolumn{8}{|l|}{ Gasoline } \\
\hline Never & 2161 & 243 & - & & 323 & - & \\
\hline Ever & 510 & 96 & 1.11 & {$[0.83-1.50]$} & 100 & 0.93 & {$[0.70-1.23]$} \\
\hline \multicolumn{8}{|l|}{ CEl } \\
\hline Low & 257 & 54 & 1.19 & {$[0.82-1.72]$} & 51 & 0.89 & {$[0.62-1.27]$} \\
\hline Medium & 205 & 32 & 1.02 & {$[0.65-1.61]$} & 46 & 1.19 & {$[0.80-1.77]$} \\
\hline High & 48 & 10 & 0.93 & {$[0.42-2.06]$} & 3 & 0.23 & {$[0.07-0.79]$} \\
\hline$p$ for trend & & & & 0.87 & & & 0.03 \\
\hline \multicolumn{8}{|l|}{ Special petroleum products } \\
\hline Never & 2439 & 300 & - & & 384 & - & \\
\hline Ever & 234 & 38 & 1.20 & {$[0.79-1.82]$} & 39 & 0.93 & {$[0.63-1.39]$} \\
\hline \multicolumn{8}{|l|}{ CEI } \\
\hline Low & 118 & 18 & 1.22 & {$[0.68-2.20]$} & 17 & 0.86 & {$[0.48-1.54]$} \\
\hline Medium & 93 & 17 & 1.21 & {$[0.65-2.24]$} & 20 & 1.07 & {$[0.61-1.89]$} \\
\hline High & 23 & 3 & 1.10 & {$[0.29-4.17]$} & 2 & 0.64 & {$[0.14-2.94]$} \\
\hline$p$ for trend & & & & 0.55 & & & 0.75 \\
\hline \multicolumn{8}{|l|}{ Diesel, fuels and kerosene } \\
\hline Never & 1753 & 175 & - & & 239 & - & \\
\hline Ever & 918 & 164 & 1.19 & {$[0.90-1.56]$} & 184 & 1.05 & {$[0.82-1.35]$} \\
\hline \multicolumn{8}{|l|}{ CEl } \\
\hline Low & 460 & 80 & 1.12 & {$[0.80-1.56]$} & 88 & 1.01 & {$[0.74-1.37]$} \\
\hline Medium & 366 & 69 & 1.36 & [0.96-1.93] & 80 & 1.21 & [0.88-1.67] \\
\hline High & 92 & 15 & 0.92 & {$[0.48-1.75]$} & 16 & 0.80 & [0.43-1.48] \\
\hline $\mathrm{p}$ for trend & & & & 0.61 & & & 0.41 \\
\hline \multicolumn{8}{|l|}{ White spirits } \\
\hline Never & 1436 & 125 & - & & 186 & - & \\
\hline Ever & 1240 & 216 & 1.14 & {$[0.82-1.58]$} & 237 & 0.93 & [0.70-1.24] \\
\hline \multicolumn{8}{|l|}{ CEI } \\
\hline Low & 620 & 93 & 1.15 & [0.80-1.67] & 112 & 0.99 & {$[0.71-1.37]$} \\
\hline Medium & 494 & 86 & 0.99 & {$[0.67-1.47]$} & 94 & 0.84 & [0.59-1.20] \\
\hline High & 126 & 37 & 1.46 & [0.88-2.43] & 31 & 0.97 & [0.58-1.60] \\
\hline$p$ for trend & & & & 0.09 & & & 0.70 \\
\hline
\end{tabular}

Abbreviations: CEI Cumulative Exposure Index

${ }^{\mathrm{a}} \mathrm{OR}$ adjusted for age at interview, residence area, alcohol consumption, smoking status, frequency and duration of smoking, exposure to asbestos 
ORs were slightly elevated for each solvent, on the order of 1.2, with no significant trend with cumulative exposure. The highest OR was found for the highest level of cumulative exposure to white spirits $(\mathrm{OR}=1$. 46; 95\% CI [0.88-2.43]), with some indication of a dose-response relationship ( $\mathrm{p}$ for trend $=0.09$ ). For laryngeal cancer, the ORs were around the null value of 1 for all solvents.

Regarding exposure to oxygenated solvents (Table 3), ever exposure to tetrahydrofuran was associated with non-significantly elevated risks of hypopharyngeal and laryngeal cancer. There was some evidence of a doseresponse relationship for hypopharyngeal cancer, with a higher OR for the highest level of cumulative exposure $(\mathrm{OR}=2.63$; $95 \mathrm{CI} \%$ [0.55-12.65]; $\mathrm{p}$ for trend $=0.07)$. We also observed a significant increased OR for laryngeal cancer risk among men with the lowest level of exposure to ethylene glycol $(\mathrm{OR}=1.75 ; 95 \% \mathrm{CI}[1.04-2.94])$ with a significant negative trend with the level of exposure ( $p$ for trend $=0.04$ ). No association appeared between exposure to other oxygenated solvents and hypopharyngeal or laryngeal cancer risk.

Further adjustment for socioeconomic status generally slightly decreased the ORs without any relevant change (see Additional file 3 for petroleum-based solvents and Additional file 4 for oxygenated solvents). Analyses considering exposure to combinations of petroleum solvents are shown in Table 4. Exclusive exposure to white spirits was associated with a non-statistically significantly elevated risk of hypopharyngeal cancer $(\mathrm{OR}=1.40 ; 95 \% \mathrm{CI}$ [0.85-2.32]), as well as other combinations including white spirits. Men exposed to the five petroleum solvents had an elevated risk of hypopharyngeal cancer $(\mathrm{OR}=2.12$; $95 \%$ CI $[0.98-4.61])$. An elevated risk of laryngeal cancer was found for exclusive exposure to diesel $(\mathrm{OR}=1.62$; 95\% CI [0.89-2.96]), but not for exposure to diesel combined with other petroleum solvents. Combined exposure to benzene, special petroleum products and white spirits was associated with a decreased risk of laryngeal cancer $(\mathrm{OR}=0.41 ; 95 \%$ CI $[0.17-1.00])$.

The analysis of exposure to combinations of oxygenated solvents (Table 5) showed that men exposed to ketones, alcohols and tetrahydrofuran had a significantly higher risk of hypopharyngeal cancer $(\mathrm{OR}=2.79 ; 95 \% \mathrm{CI}$ [1.12-6.95]); an increased OR of borderline significance was also observed for laryngeal cancer $(\mathrm{OR}=2.25 ; 95 \%$ CI [0.94-5.38]). No increased risks were found for other combinations including alcohols and ketones. No subject was exposed solely to tetrahydrofuran.

\section{Discussion}

In this study, we investigated the associations between occupational exposure to petroleum-based or oxygenated
Table 3 Association between hypopharyngeal and laryngeal cancers and occupational exposure to oxygenated solvents

\begin{tabular}{|c|c|c|c|c|c|c|c|}
\hline & \multirow{2}{*}{$\begin{array}{l}\text { Controls } \\
\mathrm{n}\end{array}$} & \multicolumn{3}{|c|}{ Hypopharynx } & \multicolumn{3}{|c|}{ Larynx } \\
\hline & & $\bar{n}$ & $\mathrm{OR}^{\mathrm{a}}$ & {$[95 \% \mathrm{Cl}]$} & $\bar{n}$ & $\mathrm{OR}^{\mathrm{a}}$ & {$[95 \% \mathrm{Cl}]$} \\
\hline \multicolumn{8}{|c|}{ Ketones and esters } \\
\hline Never & 2055 & 238 & - & & 297 & - & \\
\hline Ever & 618 & 100 & 1.01 & [0.74-1.37] & 126 & 1.10 & [0.84-1.45] \\
\hline \multicolumn{8}{|l|}{ CEI } \\
\hline Low & 309 & 46 & 1.05 & {$[0.71-1.56]$} & 62 & 1.24 & [0.88-1.75] \\
\hline Medium & 245 & 38 & 0.89 & [0.58-1.35] & 53 & 1.08 & {$[0.74-1.56]$} \\
\hline High & 64 & 16 & 1.34 & [0.70-2.59] & 11 & 0.87 & [0.42-1.79] \\
\hline$p$ for trend & & & & 0.52 & & & 0.62 \\
\hline \multicolumn{8}{|l|}{ Alcohols } \\
\hline Never & 1775 & 225 & - & & 290 & - & \\
\hline Ever & 898 & 113 & 0.95 & {$[0.72-1.27]$} & 133 & 0.90 & [0.70-1.17] \\
\hline \multicolumn{8}{|l|}{ CEI } \\
\hline Low & 447 & 45 & 0.85 & [0.59-1.25] & 64 & 0.99 & {$[0.71-1.37]$} \\
\hline Medium & 359 & 50 & 1.07 & [0.74-1.55] & 53 & 0.86 & {$[0.60-1.22]$} \\
\hline High & 91 & 17 & 1.25 & {$[0.68-2.31]$} & 16 & 0.93 & {$[0.51-1.72]$} \\
\hline$p$ for trend & & & & 0.31 & & & 0.87 \\
\hline
\end{tabular}

\section{Diethyl ether}

$\begin{array}{lllllllll}\text { Never } & 2580 & 333 & - & & 419 & - \\ \text { Ever } & 90 & 5 & 0.59 & {[0.20-1.70]} & 4 & 0.37 & {[0.12-1.11]} \\ \text { CEl } & & & & & & & \\ \text { Low } & 45 & 1 & 0.28 & {[0.04-2.19]} & 1 & 0.2 & {[0.03-1.53]} \\ \text { Medium } & 36 & 4 & 1.41 & {[0.43-4.67]} & 3 & 0.73 & {[0.20-2.65]} \\ \text { High } & 9 & 0 & - & - & 0 & - & - \\ \text { p for trend } & & & & 0.90 & & & 0.41\end{array}$

Ethylene glycol

$\begin{array}{lllllllll}\text { Never } & 2487 & 312 & - & & 387 & - \\ \text { Ever } & 183 & 26 & 0.82 & {[0.49-1.36]} & 36 & 1.00 & {[0.64-1.56]} \\ \text { CEI } & & & & & & & \\ \text { Low } & 92 & 15 & 1.08 & {[0.57-2.04]} & 28 & 1.75 & {[1.04-2.94]} \\ \text { Medium } & 72 & 11 & 0.83 & {[0.39-1.74]} & 6 & 0.39 & {[0.16-0.96]} \\ \text { High } & 19 & 0 & - & - & 2 & 0.34 & {[0.07-1.64]} \\ \text { p for trend } & & & & 0.10 & & & 0.04\end{array}$

Tetrahydrofuran

\begin{tabular}{lllllllll} 
Never & 2603 & 319 & - & & 406 & - \\
Ever & 67 & 19 & 1.67 & {$[0.87-3.21]$} & 17 & 1.39 & {$[0.73-2.63]$} \\
CEl & & & & & & & \\
Low & 35 & 7 & 1.33 & {$[0.53-3.35]$} & 9 & 1.50 & {$[0.66-3.35]$} \\
Medium & 26 & 8 & 1.57 & {$[0.61-4.08]$} & 8 & 1.62 & {$[0.65-4.07]$} \\
High & 6 & 4 & 2.63 & {$[0.55-12.65]$} & 0 & - & - \\
$\begin{array}{l}\text { p for trend } \\
\text { M }\end{array}$ & & & & 0.07 & & & 0.80 \\
\hline
\end{tabular}

Abbreviations: CEI Cumulative Exposure Index

a OR adjusted for age at interview, residence area, alcohol consumption, smoking status, frequency and duration of smoking, exposure to asbestos 
Table 4 Association between hypopharyngeal and laryngeal cancers and exposure to combinations of petroleum-based solvents

\begin{tabular}{|c|c|c|c|c|c|}
\hline \multirow[t]{2}{*}{$\begin{array}{l}\text { Exposure to petroleum-based } \\
\text { solvents }\end{array}$} & \multirow{2}{*}{$\begin{array}{l}\text { Controls } \\
n=2536 \\
n\end{array}$} & \multicolumn{2}{|c|}{$\begin{array}{l}\text { Hypopharynx } \\
n=313\end{array}$} & \multicolumn{2}{|c|}{$\begin{array}{l}\text { Larynx } \\
n=396 \\
\end{array}$} \\
\hline & & $\mathrm{n}$ & $\mathrm{OR}^{\mathrm{a}}[95 \% \mathrm{Cl}]$ & $n$ & $\mathrm{OR}^{\mathrm{a}}[95 \% \mathrm{Cl}]$ \\
\hline None & 1231 & 95 & 1 & 143 & 1 \\
\hline White spirits & 316 & 46 & $1.40[0.85-2.32]$ & 55 & $0.98[0.63-1.51]$ \\
\hline Diesel & 90 & 9 & $0.92[0.41-2.11]$ & 23 & 1.62 [0.89-2.96] \\
\hline Ben, WS & 74 & 19 & $1.55[0.79-3.03]$ & 26 & $1.27[0.70-2.29]$ \\
\hline WS, Diesel & 242 & 44 & $1.47[0.88-2.45]$ & 46 & $0.90[0.57-1.43]$ \\
\hline Ben, SPP, WS & 86 & 10 & $1.07[0.47-2.45]$ & 7 & $0.41[0.17-1.00[$ \\
\hline Gasoline, WS, Diesel & 95 & 20 & $1.48[0.75-2.90]$ & 20 & $0.93[0.50-1.73]$ \\
\hline Ben, Gasoline, WS, Diesel & 233 & 41 & $1.27[0.75-2.15]$ & 44 & 0.86 [0.54-1.39] \\
\hline All petroleum-based solvents & 55 & 15 & $2.12[0.98-4.61]$ & 14 & $1.28[0.60-2.71]$ \\
\hline
\end{tabular}

Abbreviations: WS white spirits, diesel diesel, fuels and kerosene, ben benzene, SPP special petroleum products

${ }^{\mathrm{a}} \mathrm{OR}$ adjusted for age at interview, residence area, alcohol consumption, smoking status, frequency and duration of smoking, exposure to asbestos

solvents and hypopharyngeal or laryngeal cancer. We did not find any significant excess risk when exposure to a single solvent was considered. Overall, our results do not suggest a substantial role of exposure to petroleum-based or oxygenated solvents in hypopharyngeal or laryngeal cancer risk.

Research on occupational exposure to solvents and the risk of hypopharyngeal and laryngeal cancers has been limited so far. In a case-control study conducted in Southern Europe, a significant increased risk of hypopharyngeal and laryngeal cancers was reported among men exposed to organic solvents [8]. In a multicenter case-control study in Central and Eastern Europe, exposure to organic solvents was associated with a nonsignificantly elevated risk of hypopharyngeal cancer, but no association was found with laryngeal cancer [9]. Other epidemiological studies which considered exposure to solvents in general did not report excess risks of laryngeal cancer $[9,18-21]$. Most of these studies were case-control studies, that adjusted for smoking and alcohol $[8,9,18-21]$, with the exception of a cohort study of construction workers, in which alcohol consumption was not available [21].
Exposure to solvents was assessed by industrial hygienists from detailed occupational histories in one study [9], with a JEM in three studies [8, 20, 21], and was selfreported in two studies [18, 19]. Very few studies have previously investigated exposures to specific petroleumbased and oxygenated solvents, so comparison of our results with the literature is limited.

As in our study, moderate and non-significant associations have been previously reported between laryngeal cancer and exposure to gasoline $[9,19]$ or to diesel, fuels and kerosene [9].

Our findings provide limited evidence of an increased risk of hypopharyngeal cancer in men exposed to white spirits, with elevated ORs for the highest level of cumulative exposure, and for men exposed to white spirits only or in combination with other solvents. Analogously, we previously reported non-significantly increased risks of hypopharyngeal and laryngeal cancer in women exposed to white spirits [13]. To our knowledge, only one study reported results on the relationship between exposure to mineral spirits and laryngeal cancer and found no association [9].

Table 5 Association between hypopharyngeal and laryngeal cancers and exposure to combinations of oxygenated solvents

\begin{tabular}{|c|c|c|c|c|c|}
\hline \multirow[t]{2}{*}{ Exposure to oxygenated solvents } & \multirow{2}{*}{$\begin{array}{l}\text { Controls } \\
n=2670 \\
n\end{array}$} & \multicolumn{2}{|c|}{$\begin{array}{l}\text { Hypopharynx } \\
n=338\end{array}$} & \multicolumn{2}{|c|}{$\begin{array}{l}\text { Larynx } \\
n=423\end{array}$} \\
\hline & & $\bar{n}$ & $\mathrm{OR}^{\mathrm{a}}[95 \% \mathrm{Cl}]$ & $\bar{n}$ & $\mathrm{OR}^{\mathrm{a}}[95 \% \mathrm{Cl}]$ \\
\hline None & 1684 & 207 & 1 & 268 & 1 \\
\hline Ket & 36 & 9 & $1.40[0.57-3.48]$ & 10 & $1.26[0.55-2.92]$ \\
\hline Alc, Ket & 305 & 45 & $0.95[0.63-1.43]$ & 66 & $1.15[0.81-1.63]$ \\
\hline Alc, Etg & 314 & 29 & $1.02[0.63-1.65]$ & 24 & $0.64[0.40-1.05]$ \\
\hline Alc, Ket, THF & 25 & 10 & $2.79[1.12-6.95]$ & 10 & $2.25[0.94-5.38]$ \\
\hline Alc, Ket, Etg & 158 & 23 & $0.84[0.49-1.45]$ & 27 & $0.86[0.52-1.41]$ \\
\hline
\end{tabular}

Abbreviations: OR odds-ratio, Cl confidence intervals, Ket ketones and esters, Alc alcohols, Etg ethylene glycol, THF tetrahydrofuran

${ }^{a} \mathrm{OR}$ adjusted for age at interview, residence area, alcohol consumption, smoking status, frequency and duration of smoking, exposure to asbestos 
Our findings also suggest that exposure to tetrahydrofuran may increase the risk of hypopharyngeal cancer, and to a lesser extent the risk of laryngeal cancer. For hypopharyngeal cancer, the risk increased with cumulative exposure, and a significantly elevated OR was observed among men exposed to tetrahydrofuran, ketones and alcohols. The evidence is weaker for laryngeal cancer, with lower ORs and no indication of a dose-response relationship. Tetrahydrofuran has been recently classified by the International Agency for Research on Cancer as possibly carcinogenic to humans, with sufficient evidence of carcinogenicity in animals and no data in humans [22].

Overall, the associations between hypopharyngeal cancer and exposure to tetrahydrofuran and white spirits must be interpreted with caution and need to be replicated in other studies. Little information on possible underlying mechanisms is available. Exposure to tetrahydrofuran causes liver and renal tumors in rodents [22-24]. The mechanisms are not firmly established, but are probably different from that operating in the upper airways.

The evidence of carcinogenicity of white spirits in experimental animals remains limited [25]. Studies on the genotoxicity of tetrahydrofuran and white spirits are inconclusive [23-25] and mostly negative. The upper airways are nevertheless in direct contact with inhaled toxic agents. Respiratory tract irritation following inhalation exposure has been documented in humans and laboratory animals for both tetrahydrofuran [23, 24] and white spirits [25].

Chronic irritation, inflammation and increased cell proliferation is a possible mechanism, although there are no experimental data to support this hypothesis. Such mechanisms may be relevant to other parts of the upper respiratory tract and to the lower airways. It is worth noting that exposure to mineral spirits was previously found to be associated with squamous cell carcinomas of the lung [26] and oesophagus [27]. The association between exposure to tetrahydrofuran and cancer of the respiratory tract warrants further investigation.

Another possible mechanism is that eexposure to solvents may facilitate the penetration of carcinogens through the mucosa. To further investigate this hypothesis, we evaluate the presence of multiplicative interaction between smoking, alcohol drinking, asbestos exposure and exposure to white spirits and tetrahydrofuran. No significant interaction was found, but the statistical power was limited.

Our study has some limitations. We used JEMs to assess occupational exposures retrospectively. As JEMs do not take into account the heterogeneity of tasks within the same job title, they usually generate misclassification of exposure. On the other hand, JEMs assign exposure in a reproducible and automatic way, independently of the case or control status; consequently, misclassification of exposure is likely to be non-differential.

Non-differential misclassification of exposure leads to an average bias towards the null for dichotomous exposures, and tends to disrupt dose-response trends for multilevel exposure variables. Our positive findings are therefore unlikely to be explained by exposure misclassification [28].

We did not collect information about non-occupational solvent exposure, but the relative contribution of this source of exposure is likely to be minimal. Despite an overall large number of subjects, the prevalence of exposure to some solvents was low, resulting in large confidence intervals and limited ability for in-depth analyses.

Recall bias is possible, but was limited by the use of standardized questionnaires and the average number of reported jobs was similar in cases (4.2) and controls (4.6). Selection bias is probably not a major limitation of this study: controls had a distribution by socioeconomic status and lifetime prevalence of exposure to solvents comparable to that of the general population [16]; the distribution by age, sex and cancer site of the included cases was similar to that observed in France in the same period [29]. Finally, we assessed a large number of associations, and some findings may be due to chance.

Our study has important strengths. We used data from a large population-based case-control study, with sufficient statistical power to detect moderate associations. Availability of detailed information on lifelong occupational history allowed us to assess indices of cumulative exposure and to study dose-response relationships. We adjusted for smoking, alcohol consumption, the main non-occupational risk factors, as well as occupational exposure to asbestos. In additional analyses, we also took into account socioeconomic status and other occupational exposures, therefore residual confounding is likely to be minimal.

\section{Conclusions}

This study provides weak evidence for an association between hypopharyngeal cancer and exposure to white spirits and tetrahydrofuran. Our findings do not suggest that the other petroleum-based or oxygenated solvents cause hypopharyngeal or laryngeal cancers.

\section{Additional files}

Additional file 1: Categories of exposure indices. (PDF $140 \mathrm{~kb}$ ) Additional file 2: Spearman correlation coefficients between cumulative exposures to petroleum-based and oxygenated solvents. (PDF 193 kb)

Additional file 3: Association between hypopharyngeal and laryngeal cancers and exposure to petroleum-based solvents, with adjustment for socioeconomic status. (PDF $115 \mathrm{~kb}$ ) 
Additional file 4: Association between hypopharyngeal and laryngeal cancers and exposure to oxygenated solvents, with adjustment for socioeconomic status. (PDF $116 \mathrm{~kb}$ )

\section{Abbreviations}

Alc: Alcohols; ben: Benzene; CEl: Cumulative Exposure Index; Cl: Confidence interval; diesel: Diesel, fuels and kerosene; Etg: Ethylene glycol; Ket: Ketones and esters; OR: Odds-ratio; SPP: Special petroleum products; THF: Tetrahydrofuran; WS: White spirits

\section{Acknowledgements}

The authors thank all members of the MatGéné working group from Santé Publique France and, in particular, Ms. Brigitte Dananché for providing job-exposure matrices.

Members of ICARE Study Group: Anne-Valérie Guizard (Registre des cancers du Calvados, France); Arlette Danzon, Anne-Sophie Woronoff (Registre des cancers du Doubs, France); Michel Velten (Registre des cancers du Bas-Rhin, France); Antoine Buemi, Émilie Marrer (Registre des cancers du Haut-Rhin, France); Brigitte Trétarre (Registre des cancers de l'Hérault, France); Marc Colonna, Patricia Delafosse (Registre des cancers de l'Isère, France); Paolo Bercelli, Florence Molinié (Registre des cancers de Loire-Atlantique-Vendée, France); Simona Bara (Registre des cancers de la Manche, France); Bénédicte Lapotre-Ledoux, Nicole Raverdy (Registre des cancers de la Somme, France); Sylvie Cénée, Oumar Gaye, Florence Guida, Farida Lamkarkach, Loredana Radoï, Marie Sanchez, Isabelle Stücker (INSERM, Centre for research in Epidemiology and Population Health (CESP), U1018, Environmental Epidemiology of Cancer Team, Villejuif, France); Matthieu Carton, Diane Cyr, Annie Schmaus (Inserm Epidemiologic Cohorts Unit-UMS 011 INSERM-UVSQ, Villejuif, France); Joëlle Févotte (University Lyon 1, UMRESTTE, Lyon, France); Corinne Pilorget (French Public Health Agency, Department of Occupational Health, Saint Maurice, France); Gwenn Menvielle (Sorbonne Universités, UPMC Univ Paris 06, INSERM,IPLESP UMRS 1136, Paris, France); Danièle Luce (INSERM U 1085-IRSET, Pointe-à-Pitre, France).

\section{Funding}

The ICARE study was funded by the French National Research Agency (ANR): French National Cancer Institute (INCA); French Agency for Food, Environmental and Occupational Health and Safety (ANSES); French Institute for Public Health Surveillance (InVS); Fondation pour la Recherche Médicale (FRM); Fondation de France; Fondation ARC pour la Recherche sur le Cancer; Ministry of Labour (Direction Générale du Travail); Ministry of Health (Direction Générale de la Santé).

\section{Availability of data and materials}

The data that support the findings of this study are available from the corresponding author upon reasonable request.

\section{Authors' contributions}

$\mathrm{DL}$ and $\mathrm{CB}$ designed the current study, conducted the analyses and drafted the manuscript; MC, GM and LR contributed to the statistical analysis and interpretation of the results. CP was involved in exposure assessment. SB contributed to data collection and quality control. DL and IS are the principal investigators of the ICARE study, conceived this study and coordinated the original collection of the data. All the authors critically reviewed and revised the manuscript, and gave their approval for its final version.

\section{Ethics approval and consent to participate}

Institutional Review Board of the French National Institute of Health and Medical Research (IRB-Inserm, number 01-036) and the French Data Protection Authority (CNIL number 90120). Each participant gave a written consent.

\section{Consent for publication}

Not applicable

\section{Competing interests}

The authors declare that they have no competing interests.

\section{Publisher's Note}

Springer Nature remains neutral with regard to jurisdictional claims in published maps and institutional affiliations.

\section{Author details}

'Univ Rennes, Inserm, EHESP, Irset (Institut de recherche en santé, environnement et travail)-UMR_S 1085, Pointe-à-Pitre F-97110, France. ${ }^{2}$ Univ Paris Sud, Paris Saclay University, Orsay, France. ${ }^{3}$ Département de Biométrie, Institut Curie, DRCI, PSL Research University, Paris, France. ${ }^{4}$ CESP, Cancer and Environment Team, INSERM U1018, Université Paris-Sud, Université Paris-Saclay, Villejuif, France. ${ }^{5}$ Faculty of Dental Surgery, University Paris Descartes, Paris, France. ${ }^{6}$ Sorbonne Universités, UPMC Univ Paris 06, INSERM, Institut Pierre Louis d'épidémiologie et de Santé Publique (IPLESP UMRS 1136), Paris, France. ${ }^{7}$ The French Public Health Agency, Saint Maurice, France. ${ }^{8}$ Ifsttar, UMRESTTE, UMR T_9405, Univ Lyon, Claude Bernard Lyon1 University, Lyon, France. ${ }^{9}$ Manche Cancer Registry, Cotentin Hospital,

Cherbourg-Octeville, France.

Received: 5 August 2017 Accepted: 29 March 2018

Published online: 05 April 2018

\section{References}

1. Forman D, Bray F, Brewster DH, Gombe Mbalawa C, Kohler B, Piñeros M, Steliarova-Foucher E, Swaminathan R, Ferlay J, editors. Cancer incidence in five continents, vol. X. IARC scientific publication no. 164. Lyon: International Agency for Research on Cancer; 2014.

2. Secretan B, Straif K, Baan R, Grosse Y, El Ghissassi F, Bouvard V, et al. A review of human carcinogens-part E: tobacco, areca nut, alcohol, coal smoke, and salted fish. Lancet Oncol. 2009;10:1033-4.

3. Hashibe M, Brennan P, Chuang SC, Boccia S, Castellsague X, Chen C, et al. Interaction between tobacco and alcohol use and the risk of head and neck Cancer: pooled analysis in the international head and neck Cancer epidemiology consortium. Cancer Epidemiol Biomark Prev. 2009;18:541-50.

4. Baan R, Grosse $Y$, Straif $K$, Secretan B, El Ghissassi F, Bouvard V, et al. A review of human carcinogens-part F: chemical agents and related occupations. Lancet Oncol. 2009;10:1143-4.

5. Straif K, Benbrahim-Tallaa L, Baan R, Grosse Y, Secretan B, El Ghissassi F, et al. A review of human carcinogens-part $C$ : metals, arsenic, dusts, and fibres. Lancet Oncol. 2009;10:453-4.

6. Paget-Bailly S, Cyr D, Luce D. Occupational exposures to asbestos, polycyclic aromatic hydrocarbons and solvents, and cancers of the oral cavity and pharynx: a quantitative literature review. Int Arch Occup Environ Health. 2012:85:341-51

7. Paget-Bailly S, Cyr D, Luce D. Occupational exposures and cancer of the larynx-systematic review and meta-analysis. J Occup Environ Med. 2012;54: $71-84$.

8. Berrino F, Richiardi L, Boffetta P, Estéve J, Belletti I, Raymond L, et al. Occupation and larynx and hypopharynx cancer: a job-exposure matrix approach in an international case-control study in France, Italy, Spain and Switzerland. Cancer Causes Control. 2003:14:213-23.

9. Shangina O. Occupational exposure and laryngeal and Hypopharyngeal Cancer risk in central and Eastern Europe. Am J Epidemiol. 2006;164:367-75.

10. Barul C, Fayossé A, Carton M, Pilorget C, Woronoff A-S, Stücker I, et al. Occupational exposure to chlorinated solvents and risk of head and neck cancer in men: a population-based case-control study in France. Environ Health. 2017;16:77

11. Paget-Bailly S, Guida F, Carton M, Menvielle G, Radoï L, Cyr D, et al. Occupation and head and neck cancer risk in men: results from the ICARE study, a French population-based case-control study. J Occup Environ Med. 2013;55:1065-73.

12. ICARE study group, Luce D, Stücker I. Investigation of occupational and environmental causes of respiratory cancers (ICARE): a multicenter, populationbased case-control study in France. BMC Public Health. 2011;11:928.

13. Carton M, Barul C, Menvielle G, Cyr D, Sanchez M, Pilorget C, et al. Occupational exposure to solvents and risk of head and neck cancer in women: a populationbased case-control study in France. BMJ Open. 2017;7:e012833.

14. International Labour Office. International standard classification of occupations (ISCO). Geneva: ILO; 1968.

15. Institut National de la Statistique et Des Etudes Economiques. Définitions et méthodes - Nomenclature d'activités française. Paris: INSEE; 2000.

16. Fevotte J, Dananche B, Delabre L, Ducamp S, Garras L, Houot M, et al. Matgene: a program to develop job-exposure matrices in the general population in France. Ann Occup Hyg. 2011:55:865-78.

17. Lacourt A, Leffondre K, Gramond C, Ducamp S, Rolland P, Gilg Soit Ilg A, et al. Temporal patterns of occupational asbestos exposure and risk of pleural mesothelioma. Eur Respir J. 2012;39:1304-12. 
18. Ahrens W, Jöckel KH, Patzak W, Elsner G. Alcohol, smoking, and occupational factors in cancer of the larynx: a case-control study. Am J Ind Med. 1991;20: 477-93.

19. De Stefani E, Boffetta P, Oreggia F, Ronco A, Kogevinas M, Mendilaharsu M. Occupation and the risk of laryngeal cancer in Uruguay. Am J Ind Med. 1998;33:537-42

20. Elci OC, Akpinar-Elci M, Blair A, Dosemeci M. Risk of laryngeal Cancer by occupational chemical exposure in Turkey. J Occup Environ Med. 2003;45: $1100-6$.

21. Purdue MP, Järvholm B, Bergdahl IA, Hayes RB, Baris D. Occupational exposures and head and neck cancers among Swedish construction workers. Scand J Work Environ Health. 2006;32:270-5.

22. Grosse Y, Loomis D, Guyton KZ, El Ghissassi F, Bouvard V, Benbrahim-Tallaa $L$, et al. Some chemicals that cause tumours of the urinary tract in rodents. Lancet Oncol. 2017:18:1003-4.

23. Fowles J, Boatman R, Bootman J, Lewis C, Morgott D, Rushton E, et al. A review of the toxicological and environmental hazards and risks of tetrahydrofuran. Crit Rev Toxicol. 2013;43:811-28.

24. U.S Environmental Protection Agency. Toxicological Review of Tetrahydrofuran (CAS No. 109-99-9), Document Reference EPA/635/R-11/006F, Washington, 2012; Available from: www.epa.gov/iris. Accessed 4 Aug 2017.

25. Mckee RH, Adenuga MD, Carrillo J-C. Characterization of the toxicological hazards of hydrocarbon solvents. Crit Rev Toxicol. 2015;45:273-365.

26. Siemiatycki J, Dewar R, Nadon L, Gérin M, Richardson L, Wacholder S. Associations between several sites of cancer and twelve petroleumderived liquids. Results from a case-referent study in Montreal. Scand J Work Environ Health. 1987;13:493-504.

27. Parent ME, Siemiatycki J, Fritschi L. Workplace exposures and oesophageal cancer. Occup Environ Med. 2000;57:325-34.

28. Blair A, Stewart P, Lubin JH, Forastiere F. Methodological issues regarding confounding and exposure misclassification in epidemiological studies of occupational exposures. Am J Ind Med. 2007;50:199-207.

29. Ligier K, Belot A, Launoy G, Velten M, Bossard N, Iwaz J, et al. Descriptive epidemiology of upper aerodigestive tract cancers in France: incidence over 1980-2005 and projection to 2010. Oral Oncol. 2011:47:302-7.

\section{Submit your next manuscript to BioMed Central and we will help you at every step:}

- We accept pre-submission inquiries

- Our selector tool helps you to find the most relevant journal

- We provide round the clock customer support

- Convenient online submission

- Thorough peer review

- Inclusion in PubMed and all major indexing services

- Maximum visibility for your research

Submit your manuscript at www.biomedcentral.com/submit

) Biomed Central 